\title{
Das kulturelle Gedächtnis des Sacre du printemps: Über Archaik und Moderne ${ }^{1}$
}

\section{JAN ASSMANN}

1986 veröffentlichte der Romanist Hans Robert Jauss eine kleine Schrift mit dem Titel Die Epochenschwelle von 1912. ${ }^{2}$ Im Kern handelt es sich um die Interpretation von zwei Gedichten von Guillaume Apollinaire, Zone und Lundi Rue Christine, die Ende 1912 entstanden sind und die Jauss in den Kontext ihrer Zeit und der Avantgarde des ästhetischen Modernismus ${ }^{3}$ stellt. Als Merkmale dieser neuen Kunst nennt Jauss den »Verlust der identitätsverbürgenden Anamnesis « ${ }^{4}$, also eine Krise des 1986 veröffentlichte der Romanist Hans Robert Jauss eine kleine Schrift mit dem Titel Die

1 Dieser Text geht zurück auf einen Vortrag, der ihm Rahmen des 100-jährigen Jubiläums des Sacre du Printemps gehalten wurde. Assmann, Jan: »The Cultural Memory of Le Sacre du Printemps«, in: Danuser, Hermann/Zimmermann, Heidy [Hg.]: Avatar of Modernism. The Rite of Spring Reconsidered, London: Boosey \& Hawkes 2013, S. 319-335.

2 Jauss, Hans Robert: Die Epochenschwelle von 1912. Guillaume Apollinaire: »Zone« und »Lundi Rue Christine«, Heidelberg: Winter 1986. Für Kritik und Hinweise danke ich außer den Herausgebern Renate Lachmann und Peter Gülke.

3 Ich verwende den Ausdruck »Modernismus « im Sinne des englischen »modernism«, in Bezug auf eine geistige und künstlerische Strömung der ersten Hälfte des 20. Jahrhunderts, im Unterschied zu »Moderne«/»modernity« als Epochenbezeichnung.

4 H.S. Jauss: Die Epóchenschwelle von 1912, S. 10. 
Epochenschwelle von 1912. ${ }^{5}$ Im Kern handelt es sich um die Interpretation von zwei Gedichten von Guillaume Apollinaire, Zone und Lundi Rue Christine, die Ende 1912 entstanden sind und die Jauss in den Kontext ihrer Zeit und der Avantgarde des ästhetischen Modernismus ${ }^{6}$ stellt. Als Merkmale dieser neuen Kunst nennt Jauss den »Verlust der identitätsverbürgenden Anamnesis « ${ }^{7}$, also eine Krise des kulturellen Gedächtnisses. »Tu en as assez de vivre dans l'antiquité grecque et romaine ${ }^{8}$ heißt es in Zone und die »Erfahrung der Zerstückelung des Ich in Raum und Zeit« ${ }^{9}$ also eine Krise der Subjektivität, mit der eine neue Ästhetik der Simultaneität, des ständig zerstückelnden Schnitts und der a-mimetischen Montage einhergeht, die Realitätsaspekte, Zitate und Erinnerungsfragmente einschließen kann. Die »neuartige Dunkelheit« der Texte entspringt nicht einem

»verschlüsselten oder mehrdeutigen Sinn, sondern allein dem abrupten (oft nur mit einem unvermittelten >jetzt< angezeigten) Wechsel der Erscheinungen, Visionen, Erinnerungen wie auch der sie gerade so erfahrenden Subjekte.« Zugrunde liegt eine »ästhetische Idee der Welt, die im absolut Neuen ein unvordenkliches Altes wieder erkennen lässt. ${ }^{10}$

Die engsten Parallelen zum poetischen Verfahren Apollinaires sieht Jauss im Kubismus, der Auflösung eines Gegenstands in die Vielfalt seiner verschiedenen Ansichten, in der von Picasso 1912 erfundenen CollageTechnik, die Alltagsfetzen wie Zeitungsausschnitte und andere >objets réels< in das Bild einklebt und den Ready-mades, mit denen Marcel Duchamp 1913 beginnt und die durch minimale Transposition Gegenstände

5 Jauss, Hans Robert: Die Epochenschwelle von 1912. Guillaume Apollinaire: "Zone« und »Lundi Rue Christine«, Heidelberg: Winter 1986. Für Kritik und Hinweise danke ich außer den Herausgebern Renate Lachmann und Peter Gülke.

6 Ich verwende den Ausdruck »Modernismus « im Sinne des englischen »modernism«, in Bezug auf eine geistige und künstlerische Strömung der ersten Hälfte des 20. Jahrhunderts, im Unterschied zu »Moderne«/»modernity« als Epochenbezeichnung.

7 H.S. Jauss: Die Epochenschwelle von 1912, S. 10.

8 Deutsch: »Du hast es satt, in der griechisch-römischen Antike zu leben«.

9 H.S. Jauss: Die Epochenschwelle von 1912, S. $10 \mathrm{f}$.

10 Ebd., S. $11 f$. 
der Alltagswelt in Objekte der Kunstwelt verwandeln. ${ }^{11}$ Auch für die deutsche Dichtung und Malerei bedeutete das Jahr 1912 laut Michael Hamburger einen »annus mirabilis « ${ }^{12}$ Für die Musik gilt Entsprechendes. »In der Musikgeschichte«, schreibt Jauss, »fällt die fundamentale Zäsur zwischen der klassischen Harmonielehre und der Zwölftonmusik (J.M.Hauer, A.Schönberg) - der spezifische Schnitt zur Musik der Moderne - in die Epochenwende um 1912. ${ }^{13}$

Das Jahr 1912 »äßt sich aber nicht erst aus der Rückschau auf das, was aus ihm hervorging «, als Epochenschwelle und Anbruch von etwas radikal Neuen erkennen, sondern wurde schon von »der damals hervortretenden Avantgarde des italienischen Futuristen, französischer Kubisten oder Orphisten, deutscher Expressionisten, angloamerikanischer Imagisten und russischer Kubofuturisten« als Epochenschwelle empfunden und proklamiert. ${ }^{14}$ Das hebt diese Epochenschwelle vor anderen heraus, die gewöhnlich nachträgliche Konstruktionen des kulturellen Gedächtnisses darstellen und Zäsuren in einem Prozeß schleichenden Wandels konstatieren, die den Zeitgenossen entgangen sind. Epochenschwellen haben nicht den Status historischer Ereignisse. ${ }^{15}$ Das gilt aber nicht für epochemachende Werke, deren Uraufführung oder Erscheinen im Druck Sensation machten, der Kunst eine neue Richtung wiesen, Diskurse auslösten und durchaus den Status historischer Ereignisse gewannen. In diese Reihe gehört in allererster

11 Ebd., S. 19. Vgl. auch den Marbacher Ausstellungskatalog: Neunzehnhundertzwölf. Ein Jahr im Archiv, Marbach: Dt. Schillergesellschaft 2012.

12 Hamburger, Michael: »1912«, in: Reason and Energy-Studies in German Literature, London: Routledge \& Paul 1957, S. 213-236.

13 H.S. Jauss: Die Epochenschwelle von 1912, S. 30. Die Zwölftontechnik im strengen Sinne verwendet Schönberg zwar erst ab 1923, in die Jahre um 190810 fällt aber mit dem II. Streichquartett, dessen Uraufführung 1908 in Wien einen ähnlichen Skandal auslöste wie der Sacre 1913 in Paris, und den GeorgeLiedern 1910/11 seine radikale Trennung von der Tonalität.

14 H.S. Jauss: Die Epochenschwelle von 1912, S. 25.

$15 \mathrm{Vgl}$. Gumbrecht, Hans-Ulrich/Link-Heer, Ursula [Hg.]: Epochenschwellen und Epochenstrukturen im Diskurs der Literatur- und Sprachhistorie, Frankfurt: Suhrkamp 1985; Herzog, Reinhart/Koselleck, Reinhart [Hg.]: Epochenschwellen und Epochenbewusstsein, Poetik und Hermeneutik XII, München: Fink 1987. 
Linie Strawinskys Le Sacre du printemps, der schon bei seiner Uraufführung als ein epochales Ereignis einschlug. Im Übrigen darf man natürlich diese Wende nicht auf ein einziges Jahr einschränken. Picassos Demoiselles d'Avignon entstand schon 1907, und für das Jahr 1913 gibt es sowohl eine zweibändige Dokumentation der Erscheinungen und Ereignisse europäischer Kunst und Literatur ${ }^{16}$ als auch Philipp Felix Ingolds bei weitem umfassendste und für unser Thema einschlägigste Darstellung der Epochenschwelle. ${ }^{17}$ Virginia Woolf bezeichnete »in or about December $1910 \ll$ als den Moment, wenn »human character changed, making the modern world possible for art. ${ }^{18}$ In diesen Jahren entstand auch das 1915 erschienene Buch von Carl Einstein, Negerplastik, das im Bereich der bildenden Kunst die Brücke schlug zwischen Archaik und Moderne. ${ }^{19}$ Musik und Malerei kamen sich in diesen Jahren auch näher als je zuvor. Das hängt mit dem Aufstieg des Balletts zu einem »der Katalysatoren dieser epochalen Wandlung und zu einem zentralen Feld der Kompositionsgeschichte« zusammen. ${ }^{20}$ Serge Diaghilev, der in Petersburg und Paris wirkende Impresario zog für die optische Ausstattung seiner Ballette führende Künstler heran wie Matisse, Picasso, de Chirico, Cocteau und andere und brachte auf diese Weise die Künste zusammen. So ist es denn auch alles andere als zufällig, dass Thomas Mann im Doktor Faustus das Teufelsgespräch, in dem Adrian Leverkühn der Durchbruch in völlig neue Dimensionen der Komposition angeboten wird, im Jahre 1912 stattfinden lässt. Was der Teufel als Lösung

16 Brion-Guerry, Liliane: L'annee 1913, Les Formes Esthetiques De L'oeuvre d'art a La Veille De La Premiere Guerre Mondiale: Travaux Et Documents Inedits: 1-2 (Collection D'esthetique), Paris: Klincksieck1971.

17 Ingold, Philipp Felix: Der große Bruch: Rußland im Epochenjahr 1913. Kultur, Gesellschaft, Politik. Beck: München 2000. Ich verdanke Renate Lachmann den Hinweis auf dieses Werk.

18 Köhler, S. M.: »Postmodernismus - ein begriffsgeschichtlicher Überblick «, in: Amerikastudien 22 (1977), S. 19f. zit. nach H.S. Jauss: Die Epochenschwelle von 1912 , S. 35.

19 Einstein, Carl: Negerplastik, Kurt Wolff Verlag: Berlin 1915. Zahlreiche ähnlich ausgerichtete Werke russischer Kunsthistoriker aus dieser Zeit zitiert Ingold: Der große Bruch. 2000.

20 Danuser, Hermann: Neues Handbuch der Musikwissenschaft Bd.7, Das Zwanzigste Jahrhundert, Laaber: Laaber Verlag 1984, S. 62-77. 
der Krise anpreist, an der die gesamte neuere Musik und Kunst leidet, ist der Rückgriff aufs Urtümliche: »Wir bieten Bessres, wir bieten erst das Rechte und Wahre, - das ist schon nicht mehr das Klassische, mein Lieber, was wir erfahren lassen, das ist das Archaische, das Urfrühe, das längst nicht mehr Erprobte. ${ }^{21}$ Der Sacre ist das klassische Beispiel eines Durchbruchs zum Neuen kraft der Hinwendung zum »Archaischen, dem Urfrühen $\ll$.

In dieselben Jahre fällt auch eine wissenschaftliche Beschäftigung mit dem Archaischen, die kaum weniger neuartig und bahnbrechend ist als die ästhetische und auf diese nachhaltig zurückgewirkt hat. ${ }^{22}$ Im Jahre 1912 publiziert der Soziologe Émile Durkheim sein Werk über Les formes élémentaires de la religion, in dem er die Riten der australischen Aborigines als Urformen des Religiösen untersucht. Der Philosoph Lucien Lévy-Bruhl legte 1910 mit seinem Buch Les fonctions mentales dans les sociétés inférieures eine Rekonstruktion des archaischen Denkens vor, die er später in La mentalité primitive (1922) noch ausbaute. 1913 erschien Sigmund Freuds Totem und Tabu, ein weiterer Meilenstein der Moderne, in dem Freud die Forschungen James Frazers und Robertson Smiths zur archaischen Religion mit seiner psychoanalytischen Erforschung der seelischen Archaik (die »archaische Erbschaft«) zusammenbrachte. Schon 1909 war Arnold van Genneps Werk Les rites de passage erschienen, die Grundlegung der modernen Ritualforschung. Thomas Mann hat später in seinem 1926 entstandenen »Vorspiel« zur Josephs-Tetralogie diesen doppelten Abstieg in die Zeit- und die Seelentiefe als Unterweltsfahrt beschrieben. In der Tat nehmen sowohl Frazer als auch Freud in ihren ersten, bahnbrechenden Werken auf die Unterweltsreise des Aeneas Bezug. »Der goldene Zweig " (The Golden Bough), wie Frazer sein 1890 in zwei und 1906-15 in 12 Bänden erschienenes Werk betitelte, ist der Schlüssel, der Aeneas im VI. Gesang von Vergils Aeneis das Tor zur Unterwelt öffnet. Auf dieselbe

21 Mann, Thomas: Doktor Faustus, Frankfurt: Fischerverlag 2007, S. 346.

22 Über die Wechselwirkungen zwischen Kunst und Wissenschaft in der Entdeckung des Archaischen um 1900 siehe Brandstetter, Gabriele: »Ritual as Scene and Discourse. Art and Science Around 1900 as Exemplified by Le Sacre du printemps«, in: The World of Music 40/1 (1998), S. 37-59, bes. S. 40-46. siehe auch Küster, Bärbel: Matisse und Picasso als Kulturreisende. Primitivismus und Anthropologie um 1900, Berlin: Akademie-Verlag 2003. 
Unterweltsfahrt spielt auch Sigmund Freud an, indem er seinem ersten Werk, das die neue Methode der Psychoanalyse vorträgt, der Traumdeutung (1900) einen Vers der Aeneis voranstellt: Flectere si nequeo superos Acheronta movebo »Kann ich den Himmel nicht beugen, so hetz ich die Hölle in Aufruhr «. ${ }^{23}$ Noch enger als Sigmund Freud bringt C.G. Jung Seelentiefe und Mythologie zusammen; sein entscheidendes, später überarbeitetes Buch in dieser Richtung erschien 1912 unter dem Titel Wandlungen und Symbole der Libido. Die Aufdeckung der archaischen, von Christentum und Aufklärung überlagerten Elemente in den Mythologien der europäischen Völker verstand sich im Licht der Psychoanalyse als eine Erkundung des kollektiven Unbewussten.

In einer Rezension des 1922 erschienenen Ulysses von James Joyce bringt T.S. Eliot im Jahre 1923 rückblickend diese spezifisch moderne oder modernistische Hinwendung zum Archaischen auf den Begriff der »mythischen Methode « und deutet sie als eine Reaktion auf die allgemeine Orientierungslosigkeit einer ihrer Traditionen entfremdeten Zeit:

»In der Verwendung des Mythos und der durchgängigen Parallelisierung von Jetztzeit und Altertum verfolgt Joyce eine Methode, die andere nach ihm verfolgen werden müssen. [...] Es handelt sich um ein Verfahren, dem immensen Panorama von Vergeblichkeit und Anarchie, als das sich uns die Zeitgeschichte darstellt, Form und Sinn zu geben. [...] Psychologie, Ethnologie und [J. Frazers] The Golden Bough kamen zusammen, um etwas möglich zu machen, was wenige Jahre zuvor noch unmöglich schien. Anstelle der narrativen Methode sollten wir jetzt die mythische Methode verwenden. $\ll^{24}$

23 Vergil, Aeneis VII 312 (Übers. Johannes Götte, München: Heimeran 1955).

24 »In using the myth, in manipulating a continuous parallel between contemporaneity and antiquity, Mr. Joyce is pursuing a method which others must pursue after him. [...] It is simply a way of controlling, of ordering, of giving a shape and a significance to the immense panorama of futility and anarchy which is contemporary history. [...] Psychology, ethnology, and The Golden Bough have concurred to make possible what was impossible even a few years ago. Instead of narrative method, we may now use the mythical method.« Eliot, Thomas Stearns: »Ulysses, Order, and Myth«, in: The Dial, (11/1923) abgedr. in: Frank Kermode (Ed.): Selected Prose of T.S. Eliot, London: Faber \& Faber 1975, S. 175-178. Frick, Werner: >Die Mythische Methode`. Komparatistische Studien 
Lange vor Joyce, in unmittelbarer zeitlicher und räumlicher Nähe Strawinskys, praktizierte Andrej Belyi die smythische Methode< in seinem Roman Petersburg, der gleichzeitig mit Le Sacre 1911-1913 entstand. ${ }^{25}$ Auf ihre Weise folgen dieser Methode auch Igor Strawinsky, sein Librettist und Bühnenbildner Nikolai Roerich und sein Choreograph Vaclav Nijinsky in der Schöpfung des Sacre du printemps.

\section{Le Sacre du Printemps und die ERfindung DES PRimitiven}

Der Rückgriff auf die Archaik hat ein doppeltes Gesicht. Auf der einen Seite lässt er sich als ein romantisches Projekt verstehen, das auf den Spuren Herders die ursprünglichsten Lieder und Bräuche der Völker sammelt, und auf der anderen Seite als ein avantgardistisches Projekt, das gegen die Seelenlosigkeit einer der Natur entfremdeten Zeit revoltiert und mit seiner Rückkehr zum Ursprung einen »Auszug aus der entzauberten Welt ${ }^{26}$ inszeniert. »Interessante Lebenserscheinungen«, verallgemeinert Thomas Mann, »haben wohl immer dies Doppelgesicht von Vergangenheit und Zukunft, wohl immer sind sie progressiv und regressiv in einem. ${ }^{27}$ Der romantische Aspekt ist von einer nostalgischen Sehnsucht nach einer verlorenen Ganzheit bestimmt, dem modernistischen geht es dagegen um die abgründige Fremdheit und Grausamkeit des Archaischen. Im Sacre du printemps kommt beides zusammen. Roerichs Szenario steht eher auf der

zur Transformation der griechischen Tragödie im Drama der klassischen Moderne, Tübingen: Niemayer 1998. Zu Eliot speziell S. 213-223.

25 Siehe hierzu Lachmann, Renate: Gedächtnis und Literatur, Frankfurt a.M.: Suhrkamp 1990, S. 88-125; in diesem Roman wird »der Petersburgmythos, als Basismythos, in einer Vielzahl anderer Mythen >gespiegelt< und werden die narrative und die stilistische Ebene des Romans von anderen narrativen und stilistischen Formationen mitgeprägt.«, S. 93.

26 So der Titel eines Buches von Norbert Bolz, der die hier gemeinte Sache genau trifft, sich aber auf etwas Anderes bezieht, vgl. Bolz, Norbert: Auszug aus der entzauberten Welt. Philosophischer Extremismus zwischen den Weltkriegen, München: Fink 1989.

27 Mann, Thomas: Doktor Faustus, S. 282f. 
romantischen, Strawinskys Musik, radikal und zuweilen brutal, auf der modernistischen Seite. ${ }^{28}$

Roerichs antiquarisches Interesse am heidnischen russischen Altertum, die Suche nach einem wahren, vorchristlichen, »wilden« Russland hat etwas durchaus Romantisches, und die wichtigste Quelle, die er benutzt und Strawinsky empfiehlt, Alexander Afanassjews (1826-1871) dreibändiges Werk über »Die poetische Einstellung der Slaven zur Natur" (Poèticheskiye vozzreniya slavian na prirodu, 1866-69) gehört eindeutig in die romantische Tradition. ${ }^{29}$ Roerichs Verbindung von Neopaganismus und Neonationalismus erinnert nicht zufällig an gleichgerichtete Bewegungen in Deutschland, vor allem die Münchner Kosmiker. Auch die Wiederkehr des heidnischen Kosmotheismus um die Jahrhundertwende ist eine Reaktion auf die Krise der Moderne. ${ }^{30}$ Hier suchte man Heilung von der »hoffnungslosen Fragmentierung und Isolierung « des »der Erde entfremdeten modernen Menschen«, wie es der Avantgardist Alexander Blok, ein Freund Nikolai Roerichs, in einem Essay mit dem bezeichnenden Titel The poetry of Magic and Spells (1908) ausdrückte. ${ }^{31}$ Dem russischen Neoprimitivismus, der 1913 seinen Höhepunkt erreichte, ging es darum, »den vorkulturellen, vorkünstlerischen Nullpunkt zu eruieren, an dem Natur und Kultur, Leben

28 Taruskin, Richard: „A Myth of the Twentieth Century: The Rite of Spring, the Tradition of the New, and , The Music Itself « in: Modernism/Modernity 2.1, (1995), S. 1-26, betont etwas einseitig den romantischen Aspekt des Sacre und die Kontinuität des Projekts mit dem 19. Jh. und verlegt den eigentlichen Bruch in die frühen 1920er Jahre, als sich Strawinsky selbst energisch von den romantisch-folkloristischen Aspekten des Balletts absetzte und die Modernität der »Musik selbst« betonte.

29 Sehr viel bekannter und auch ins Deutsche übersetzt ist Afanassjews Märchensammlung Narodnye russkie skazki, Moskau 1855-1863, aufgrund derer A. als >der russische Grimm^ gilt. Zu N. Roerich siehe Decter, Jacqueline: Nicholas Roerich. Leben und Werk eines russischen Meisters, Basel: Sphinx 1989.

30 Assmann, Aleida: »Pan, Paganismus und Jugendstil«, in: Hans-Joachim Zimmermann [Hg.], Antike Tradition und neuere Philologien. Symposium zu Ehren des 75. Geburtstags von Rudolf Sühnel, Heidelberg: Winter 1984, S. 177-195.

31 Zitiert nach Taruskin, Richard: Stravinsky and the Russian Traditions. A Biography of the Works through Mavra, 2 Bde., Oxford: Oxford University Press 1996, Bd. I, S. 849. 
und Kunst, noch nicht getrennt, sondern im kollektiven mythischen Bewusstsein eins waren. $\ll^{32}$

Doch geht nicht nur die Vorstellung einer nostalgisch verklärten, sondern auch die einer fremden und grausamen Archaik auf die Romantik zurück. Hier ist der Name Johann Jakob Bachofens zu nennen. Er war der Vertreter einer dreistufigen Kultur- Evolutionstheorie, die Hetärismus, Matriarchat und Patriarchat heißen oder das tellurische, lunare und solare, also erdhafte, mondhafte und sonnenhafte Zeitalter. In der tellurischen, erdverbundenen Phase »herrscht der Todesgedanke über den des Zeugens und Lebengebens vor«. Dahin gehört auch das Menschenopfer. Die biblischen Erinnerungsspuren an die Opferung alles Erstgeborenen verbinden sich mit dem Frühjahrsfest der Aussaat. In diesem Vorstellungskreis des Tellurischen oder Chthonischen bewegt sich auch die nach eigener Aussage auf eine Art Tagtraum Strawinskys während der Arbeit am Feuervogel zurückgehende Idee vom Todestanz des geopferten Mädchens ${ }^{33}$ und vor allem Nikolai Roerichs Ausarbeitung dieser Idee zu einer Bilderfolge von Frühlingsriten »aus dem heidnischen Russland«. Bachofen rekonstruiert oder vielmehr phantasiert die tellurische Phase mit sehr viel Liebe und Einfühlungsvermögen, aber als eine zu überwindende Kulturstufe. Er postuliert, auch darin ein Kind seiner Zeit, die Dynamik des Fortschritts, der die kulturelle Entwicklung antreibt zu höheren Stufen, vom Erdhaften zum Mondverbundenen und schließlich Sonnenhaften. Andererseits aber betont er die gegenläufige Dynamik des Rückfalls, von der der Fortschritt ständig bedroht ist. Diese Tendenz und Gefahr einer Regression in den Hetärismus sieht Bachofen in der Gestalt des Gottes Dionysos verkörpert. Dionysos ist bei Bachofen ein Gott des Rauschs und der Sinnenlust; eine »Heimsuchung « und ein Rückfall in den längst überwundenen Kulturzustand des Hetärismus. ${ }^{34}$ Bei Nietzsche erscheint dann in der Geburt der Tragödie (1872) mit ihrer zukunftsweisenden Engführung von Ritual und Musik

32 P.F. Ingold: Der große Bruch, S. 180f.

33 Roerich kommt wohl (trotz eigener Aussage) als Erfinder des Motivs nicht in Betracht, weil er zu sehr dem Ideal archäologischer Authentizität verpflichtet war, um auf ein so romantisch-imaginatives Ritual wie den Todestanz eines erwählten Mädchens zu verfallen.

34 In eine ähnliche Richtung wird dann, worauf unten noch eingegangen wird, Adornos Kritik am Sacre gehen. 
gerade der Kult des Dionysos als die Urszene der abendländischen Kunst und das Archaische als Quelle schöpferischer Erneuerung.

Für die russische Avantgarde repräsentieren die Skythen, dieses asiatische Reitervolk, das durch reiche Bodenfunde vom 9. vorchristlichen bis zum 3. nachchristlichen Jahrhundert im Gebiet der heutigen Ukraine bezeugt ist und schon die Phantasie der Griechen stark beschäftigt hat, die tellurische, todes- und fruchtbarkeitsverbundene Archaik, stammen doch so gut wie alle Funde (von oft atemberaubender Schönheit und Kostbarkeit) aus Grabhügeln (Kurganen). Skivstvo, »Skythianismus« nennt sich die Kunstrichtung eines »neuen Barbarismus $\aleph^{35}$ als deren prominentester Vertreter auf musikalischer Seite der junge Sergej Prokofjew mit seiner Skythischen Suite (1915) hervortritt. Ein anderes Ideal des modernistischen Archaismus, das in Roerichs und Strawinskys Konzeption des Sacre zum Ausdruck kommt, ist >Stichiya<, Unmittelbarkeit, d.h. Präsentation anstatt Repräsentation, Vollzug anstatt Erzählung. Darauf wird im zweiten Teil dieser Studie im Zusammenhang der Gedächtnisbeziehung von Musik noch zurückzukommen sein. Roerich und Strawinsky ging es um »eine unmittelbare Darstellung des Altertums ohne ein bestimmtes dramatisches Thema«. Was sie zeigen wollten, war keine Geschichte (»there is no plot«), sondern ein Mysterium: »the mystery of the great upsurge of creative force of spring.« Daher sollte aus der Choreographie jede Form narrativer Pantomime verbannt werden, wie sie im Feuervogel noch eine so bedeutende Rolle spielte. ${ }^{36}$ $\gg$ Frühlingsopfer< ist kein $>$ Ballett ««, notierte denn auch Sergej Wolkonskij. »Es ist ein Ritual, eine altertümliche kultische Handlung. ${ }^{37}$

35 R.Taruskin: Stravinsky and the Russian Traditions, S. 855.

36 R.Taruskin: Stravinsky and the Russian Tradition, S. 865. Natürlich ist Gabriele Brandstetter (s. Anm. 12) darin Recht zu geben, dass es sich beim Sacre nicht um den Vollzug, sondern die Repräsentation eines Rituals handelt, aber - das macht sie vollkommen deutlich - auf keinen Fall im Medium des klassischen Balletts, dessen Formensprache in V. Nijinskys Choreographie auf den Kopf gestellt und zerstört wird, um dem Ausdruck des Wilden und Primitiven Raum zu geben.

37 Fürst Sergej Wolkonskij: »Die Ballets russes in Paris«, zit. nach P.F.Ingold, Der große Bruch, 562. Natürlich ist Gabriele Brandstetter (s. Anm. 13) darin Recht zu geben, dass es sich beim Sacre nicht um den Vollzug, sondern die Repräsen- 
Modelle seiner Tableaux de la Russie païenne suchte Roerich aber weniger bei den Skythen als vielmehr in der Folklore vor allem der nördlichen und westlichen Peripherie Russlands, in Litauen und Weißrussland, wo sich mehr Survivals heidnischer Riten und Bräuche erhalten haben als im intensiv christianisierten >heiligen Russland s selbst. Das Frühlingsfest Semik und das Fest der Johannisnacht Kupala werden noch heute mit rituellen Chortänzen, sogenannten chorovod gefeiert, wie sie auch im Sacre vorkommen. Ursprünglich hatten Roerich und Strawinsky auch an eine Johannisnachtfeier gedacht. Erst später wurde von Sommer auf Frühling umgeschaltet, wenn die Kräfte der Erneuerung und Fortpflanzung erwachen. In dieser Phase heißt die Sonne yarilo, von yariy "glühend «. Afanassjew beschreibt das Wesen des Gottes dieser Sonnenphase als

»frühlingshaftes Licht und Wärme, junge, heftige, gewaltsam erwachende Kräfte, erotische Leidenschaft, Wollust und Befruchtung - Vorstellungen, die untrennbar mit den Manifestationen des Frühlings und seiner erschreckenden Phänomene verbunden sind $\ll^{38}$

Das Alter dieser Bräuche wird durch die mittelalterlichen Chroniken bezeugt, die sie aus kirchlicher Sicht aufs Heftigste verdammen. Diese Menschen, heißt es in der sprimary chronicles»lebten in den Wäldern wie die Tiere ... es gab keine Hochzeiten zwischen ihnen, sondern nur Spiele zwischen den Dörfern. Wenn sich die Leute zu Spielen, Tänzen und allen anderen teuflischen Lustbarkeiten versammelten, raubten sich die Männer

tation eines Rituals handelt, aber - das macht sie vollkommen deutlich - auf keinen Fall im Medium des klassischen Balletts, dessen Formensprache in V. Nijinskys Choreographie auf den Kopf gestellt und zerstört wird, um dem Ausdruck des Wilden und Primitiven Raum zu geben.

38 Afanasyev, Alexander N.: Poèticheskiye vozzreniya slavyan na prirodu, 3 vols. Moskau: Sovremennyj Pisatel' 1995, S. 1865-69 nach R. Taruskin: Stravinsky and the Russian Traditions, S. 882: »vernal light and warmth, youthful, impetuous, violently awakening forces (of sexuality), erotic passion, lasciviousness and fecundation: ideas inseparable from the manifestations of spring and its terrifying phenomena.« 
ihre Frauen. ${ }^{39}$ Diese Stelle liegt im Sacre den Nummern $>$ Jeux des cités rivales $<$ und Jeu du rapt< zugrunde und beschreibt genau, was Bachofen unter Hetärismus versteht.Ein Menschenopfer ist in diesem Zusammenhang jedoch nicht bezeugt. Yurij Sokolov erwähnt die Verbrennung einer

"zeremoniellen Strohpuppe, die auch durch einen Baum, eine Birke ersetzt werden kann. Manchmal, vor allem in der Ukraine, wird die zentrale Rolle auch von einem Mädchen gespielt, das mit einer Girlande geschmückt ist. Um sie herum werden Chortänze (khorovod) aufgeführt und zu ihren Ehren Lieder gesungen. ${ }^{40}$

Afanassjev beschreibt ähnliche Bräuche aus Weißrussland im Zusammenhang des Johannisfests Kupala.

»In der Morgendämmerung des Johannistages wählen die Bauernmädchen die Schönste aus ihrer Mitte, ziehen sie nackt aus und umwinden sie von Kopf bis Fuß mit Girlanden. Dann machen sie sich in den Wald auf, wo Dzevko-Kupalo (wie die Erwählte genannt wird) Kränze verteilen muss, die im voraus geflochten wurden. Sie muss sich blind an diese Aufgabe machen, während sie ein fröhlicher chorovod der Mädchen umringt. Auf der Grundlage der Kränze werden Vorhersagen getroffen: ein grüner Kranz bedeutet ein reiches und verheiratetes Leben, ein verwelkter Kranz sagt Armut und unglückliche Heirat voraus. $\ll^{41}$

39 »living in the forests like the very beasts ...there were no marriages between them but simply games between the villages. When the people gathered for games, for dancing, and for all other devilish amusements, the men on these occasions carried off wives for themselves...« (Primary Chronicle), nach R. Taruskin: Stravinsky and the Russian Traditions, S. 884.

40 Sokolov, Yurij: Russian Folklore, Hartboro, PA: Folklore Associates 1966 (Russkij fol'klor, Moskau 1938), S. 196.

41 "Among the Byelorussians, with the dawning of St Johns Day, the peasant girls choose the most beautiful maiden from their midst, strip her naked, and wind her round with fine garlands from head to toe. Then they set off for the forest, where Dzevko-Kupalo (for that is what the chosen maiden is called) must distribute wreaths among her friends, which have been prepared in advance. She sets about this task blindfolded, while around her a merry maiden's khorovod starts up. Auguries are made on the basis of who gets which wreath. A living wreath vouchsafes a rich and happy married life, while a dead, withered one for- 
Diese Stelle ist wohl das Vorbild für die >augurs printanières < und die >glorification de l'Élue ‘. An diesen und vielen anderen Beispielen zeigt sich Roerichs Bemühen um archäologisch-ethnographische Authentizität seiner Bilder aus dem heidnischen Russland. In seinem Werk verbanden sich die künstlerische und die wissenschaftliche Beschäftigung mit dem >Urfrühen<, wie sie für die Epochenwende um 1912 insgesamt charakteristisch ist.

\section{MusIK UND GEDÄCHTNIS}

Die Musik unterhält als Zeitkunst vielfältige Beziehungen zum Gedächtnis, von denen ich in diesem Zusammenhang drei herausgreifen möchte: (1) Die Rolle der Erinnerung in der Organisation des musikalischen Kunstwerks, (2) seine Formen des Verweises auf Vergangenheit außerhalb seiner und (3) sein Nachleben im kulturellen Gedächtnis. Was den ersten Aspekt angeht, entwickelt ein musikalisches Werk von einigem Umfang gewissermaBen sein eigenes Gedächtnis, indem es sich in seinem Verlauf in verschiedenen Formen wörtlicher oder variierender Wiederaufnahme serinnert< an das, was vorherging. Die Musik konstituiert in ihrem Verlauf einen Erinnerungsraum, in dem Ereignisse Folgen haben, Motive gleichsam Schicksale durchmachen, sich etwas entwickelt und auflöst. Natürlich geht es hier immer um die Wahrnehmung und das Gedächtnis des Zuhörers und nicht der Musik selbst. Dabei handelt es sich aber um den impliziten, also dem Kunstwerk immanenten und seine Form und Struktur mitbestimmenden Zuhörer. Diese Gedächtnisbezogenheit gilt für alle Zeitkunst, für das musikalische Kunstwerk aber noch in anderem Sinne als das sprachliche, da hier die Möglichkeiten semantischer Referenz weitgehend entfallen. Daher entwickelt sie Formen der Selbstreferentialität, die in diesem Umfang der Dichtung fremd sind. So wird die Gedächtnismetapher dem musikalischen Kunstwerk sowohl hinsichtlich seines Zeitaspekts als auch seiner inneren Semiose gerecht, seinen Möglichkeiten, durch Rückbezüge und interne Anspielungen Bedeutung zu erzeugen und ein Gedächtnis seiner eigenen Vergangenheit auszubilden, eine Art von Identität, die durch die verschie-

tells poverty and an unfortunate marriage.« (Afanassjew Bd. 3, S. 723 nach R. Taruskin: Stravinsky and the Russian Traditions, S. 885). 
denen Stadien seiner Entwicklung eine ästhetische Form und Einheit erzeugt.

Die Form von Erinnerung, die Strawinsky im Sacre bevorzugt anwendet, ist der Verweis auf Kommendes, der >annoncierte Auftritt< von Themen und Motiven. So erklingt z.B. ein Motiv, das fast die ganzen augures printaniers hindurch wiederholt wird, bereits in der vorhergehenden Introduktion und wird dort in den Takten 4f. und 7-9 nach [12] von den 1. Vl. pizzicato vorgetragen, bevor es dann in dem folgenden Stück nach 16 Takten wieder auftritt und ostinat wird. In gleicher Weise hat, um ein weiteres Beispiel zu nennen, das charakteristische Thema in parallelen Terzen, das die Posaunen in [28] Takt 5 erstmals anstimmen (bis [30]) erst im nächsten Stück (rondes printanières) ab [50] Takt 3 seinen eigentlichen Auftritt, der sich dann ab [53] geradezu triumphal durchsetzt und auch in dem anschlieBenden jeu des cités rivales in [60]-[62] sowie [64]f. nachklingt. Auch das Thema der cercles mystérieux [91]ff. erklingt bereits in der Introduktion zum II. Teil 2 Takte vor [82] (und dann in [83], [84], [89]). In dem Netz zahlreicher derartiger Vor- und Rückverweise konstituiert sich die Erinnerung und damit die Identität des Werks. Gerade diese gedächtnisförmige Identität nun hat Th. W. Adorno dem Sacre abgesprochen:

»Solche Identität zu umgehen ist eines der primären Anliegen von Strawinskys Technik archaisch-musikalischer Bilder. Gerade weil jedoch das Motiv selber noch nicht $>$ da $<$ ist, werden die verschobenen Komplexe immerzu wiederholt, anstatt daß aus ihnen, wie es in Schönbergs Terminologie heißt, Konsequenzen gezogen wären. Der Begriff der dynamischen musikalischen Form, welcher die abendländische Musik von der Mannheimer bis zur gegenwärtigen Wiener Schule beherrscht, setzt eben das als identisches, geprägtes festgehaltene, ob auch unendlich kleine Motiv voraus. Seine Auflösung und Variation konstituiert sich einzig gegenüber dem in Erinnerung bleibend Bewahrten. Musik kennt nur um so viel Entwicklung, wie sie ein Festes, Geronnenes kennt. Die Strawinskysche Regression, die dahinter zurückgreifen möchte, ersetzt eben darum den Fortgang durch die Wiederholung. [...] Strawinsky unterscheidet sich vom subjektiv-dynamischen Prinzip der Variation eines eindeutig Gesetzten durch eine Technik permanenter Ansätze, die vergeblich gleichsam nach dem tasten, was sie in Wahrheit nicht erreichen und nicht leisten 
können. Seine Musik weiß von keiner Erinnerung und damit von keinem Zeitkontinuum der Dauer. Sie verläuft in Reflexen. $\ll^{42}$

Was Adorno hier im Blick hat, ist etwas anderes als das, was ich oben als eine Form musikalischer Erinnerung beschrieben habe. Dieser Unterschied lässt sich vielleicht anhand der Begriffe $>$ Erinnern, Wiederholen, Durcharbeiten< verdeutlichen, die Freud als Beitrag zur psychoanalytischen Behandlungstechnik eingeführt hat. ${ }^{43}$ In Adornos Einschätzung herrscht im Sacre nur Wiederholen, aber kein Erinnern und Durcharbeiten. Für ihn steht »Strawinskys Schockmusik unter Wiederholungszwang $«{ }^{44}$ Zweifellos spielt im Sacre >Wiederholen<, der erinnerungslose Puls der irregulären Rhythmen und der ständig wiederholten ostinaten $>$ Zellen<, die dominierende Rolle. Es gibt aber auch Themen, die sich über 4-8 Takte entfalten und eine durchaus distinkte Gestalt gewinnen, echte, sangbare und durch ihr mehrfaches Auftauchen eindringliche Melodien, die sich im Gedächtnis des Hörers festsetzen und jedem nach dem Erlebnis des Sacre in Erinnerung bleiben. Doch würde Adorno auch dies unter >Wiederholen< subsumieren. Was er vermisst und dem Stïck als »Erinnerungslosigkeit« vorwirft, ist offenkundig das Element >Durcharbeiten<. Was man darunter verstehen könnte, macht Adorno im Zusammenhang der These klar, Strawinsky würde im Sacre die Zeit verräumlichen, den »temps espace gegen den temps durée ausspielen. $\ll^{45}$ Diese $»$ Verfahrensweise $[\ldots]$ macht sich zum Anwalt von Rationalisierung im Sinn erinnerungsloser Meß- und Zählbarkeit. $\ll^{46}$ Musik »wird zum Parasiten der Malerei degradiert.« Musik, »die Zeit in sich aufnimmt « kennt demgegenüber »den Übergang, die Steigerung, den Unterschied von Spannungs- und Auflösungsfeld, von Exposition und Fortsetzung, von Frage und Antwort $\ll^{47}$ Das Ideal des Durcharbeitens im

42 Adorno, Theodor W.: Philosophie der neuen Musik, Frankfurt a.M.: Suhrkamp 1978, S. 131.

43 Freud, Sigmund: »Erinnern, Wiederholen, Durcharbeiten« (1914), Studienausgabe, Ergänzungsband, Schriften zur Behandlungstechnik, Frankfurt 1982, 207215.

44 Th. W. Adorno, Philosophie der neuen Musik, S. 163.

45 Th. W. Adorno, Philosophie der neuen Musik, S. 176.

46 Ebd., S. $176 f$.

47 Ebd., S. 178. 
Sinne motivischer Arbeit und entwickelnder Variation verlegt das Fortschrittsideal in das Zeitkunstwerk selbst: es soll nicht nur über seine Vorgänger, sondern auch innerhalb seines eigenen Verlaufs über seine Anfänge, die Exposition, fortschreiten. Durcharbeiten bedeutet für Adorno die Verabsolutierung der $>$ Durchführung $<$, jenes Elements des klassischen Sonatensatzes, das bei Beethoven »zum Zentrum der gesamten Form « wird. Bei Brahms schließlich "gibt es nichts Unthematisches mehr«. Schönberg nimmt diese »Beethoven-Brahmsische Tendenz « auf und »kann das Erbe der klassischen bürgerlichen Musik beanspruchen ${ }^{48}{ }^{48}$ Darin sieht Adorno die normative Form, wie Musik Zeit in sich aufzunehmen hat.

Diese Form der Zeit, Entwicklungszeit, Fortschrittszeit, ist zweifellos genau das, was Strawinsky im Sacre destruieren und vermeiden wollte, mit derselben Konsequenz wie die avantgardistische Malerei sich von der Zentralperspektive und der avantgardistische Roman (Petersburg, Ulysses) von der linearen Narrative emanzipierten. ${ }^{49}$ Daher gibt es im Sacre zwar über die ostinate Wiederholung hinaus auch erinnerungsstiftende Wiederaufnahmen von Themen über größere Abstände hinweg, das muß gegen Adorno betont werden, aber, und darin wird man Adorno Recht geben, ohne variierende Entwicklung und Durchführung dieser Themen. Was Strawinsky anstrebt, ist Zeit als Gegenwart: »Die Musik«, schreibt er in seinen Erinnerungen (1936),

wist der einzige Bereich, in dem der Mensch die Gegenwart realisiert. Durch die Unvollkommenheit unserer Natur unterliegen wir dem Ablauf der Zeit, den Kategorien der Zukunft und der Vergangenheit, ohne jemals die Gegenwart >wirklich machen zu können, also die Zeit stillstehen zu lassen. ${ }^{50}$

Das entspricht genau dem Prinzip der Stichiya, von dem oben die Rede war: Herstellung, nicht Darstellung. Vollzug, nicht Erzählung. Der pro-

48 Ebd., S. 57-59.

49 Vgl. aber Vlad, Roman: »Reihenstrukturen im Sacre du Printemps«, in: Metzger, Heinz-Klaus/Riehn, Rainer [Hg.]: Igor Strawinsky, Musik - Konzepte 34/35, München: edition text+kritik 1984, S. 4-64, der das gesamte Werk als Entfaltung einer Keimzelle, des »ostinaten « Motivs des-b-es-b erklärt.

50 Igor Strawinsky. Leben und Werk - von ihm selbst, Zürich: Atlantis-Verlag und Mainz: Schott 1957, S. 59. 
grammatischen Flächigkeit der modernen Malerei entspricht die >Räumlichkeit< von Strawinskys Musik mit dem gleichen Effekt der Eskamotierung des Subjekts als dem Bezugspunkt sowohl der Perspektive als auch der Entwicklungszeit. Im Sacre tritt dies Prinzip so besonders deutlich hervor, weil es im Dienst der Idee steht, das Archaische, das >Urfrühe $<$ so sinnfällig wie möglich zu beschwören.

Darin liegt die zweite Form der Beziehung zwischen Musik und Zeit. Ein Musikwerk kann nicht nur an seine eigene »Vergangenheit«, sondern auch an andere Musik erinnern. Hier kommt nun die Archaik ins Spiel in Form der oft nur bruchstückhaft zitierten Volksmelodien und Motive, die Strawinsky verwendet, um das »heidnische Russland « musikalisch erstehen zu lassen. Strawinsky verwendete sie aber nicht als Zitate, sondern nach Kräften und bis zur Unkenntlichkeit anverwandelt und integriert in seine durchaus neue Musik als eine Art imaginäre Volksmusik. ${ }^{51}$ Das oben als Beispiel für Wiederholung erwähnte »ostinate Motiv«, das in den augurs printaniers über hundertmal auftritt und schon in der Introduktion anklingt, geht offensichtlich, wie Roman Vlad gezeigt hat, auf das Lied der Wolgaschiffer zurück, das Strawinsky dem postzaristisch-präbolschewistischen Russland als Staatshymne vorschlug. ${ }^{52}$ Zur Evokation des Archaischen greift Strawinsky neben russischen Volksliedern, die er vermutlich im Kopf hatte, vor allem auf litauische und weißrussische Melodien aus der (ihm wohl von Roerich empfohlenen) Sammlung von Anton Juszkiewicz zurück. $^{53}$ Diese Anleihen an der Folklore sind von Taruskin ${ }^{54}$, Hill ${ }^{55}$ und anderen sorgfältig identifiziert worden und müssen hier nicht noch einmal behandelt werden. Das Interessante dieser Verwendung folkloristischen Materials ist aber, dass Strawinsky in späteren Jahren, in seiner neoklassischen Periode, in der er sich von seinen russischen Werken abgewandt hat, davon nichts mehr wissen wollte und nur das Fagott-Motiv der Introduktion

51 Hill, Peter: Stravinsky: The Rite of Spring, (= Cambridge Music Handbooks, Bd. 11), Cambridge: Cambridge University Press 2000, S. 114.

52 Vlad, a.a.O., 16-18. Vlad zitiert das Lied in einem eigenen Klavierauszug nach Strawinskys Arrangement für Bläser und Schlagzeug (1917).

53 Juszkiewicz, Anton und Badouin de Courtenay, Jan N.: Melodje ludowe litewskie, Krakau 1900.

54 R. Taruskin: Stravinsky and the Russian Tradition, S. 891-966.

55 P. Hill; Stravinsky: The Rite of Spring, S. 35-39. 
als Anleihe an genuiner (in diesem Fall litauischer) Volksmusik gelten ließ.$^{56}$ Offenbar lag ihm daran, jede Assoziation mit dem russischen Neopaganismus und Neonationalismus zu vermeiden wie auch mit der spätromantischen russischen Musik und ihrer exzessiven Verwendung volkstümlicher Motive. Dass das "russische Heidentum « irgendetwas mit der ursprünglichen Idee des Sacre zu tun haben könnte, wurde von Strawinsky seit 1920 heftig abgestritten. ${ }^{57}$ Die Musik sei zuerst dagewesen; das heidnische Setting sekundär. In Wahrheit ist im 20. Jahrhundert kaum eine andere Musik, es sei denn von Strawinsky selbst, geschrieben worden, die stärker und eindeutiger vom Geist der urtümlich russischen Musik geprägt ist, was auch in Les Noces deutlich wird. Etwas Entsprechendes, ihre Sättigung, sozusagen, mit dem Gedächtnis der verschiedensten Musiktraditionen, gilt für das gesamte Euvre Igor Strawinskys und verbindet seine russische mit seiner neoklassischen und allen späteren Perioden seines Schaffens. Nie ist eine gedächtnis-gesättigtere Musik geschrieben worden. Strawinsky, überliefert Pierre Boulez, habe immer gesagt, dass für ihn die Tradition das Entscheidende sei und dass er die Tradition fortführe. ${ }^{58}$ Tradition ist hier nicht im akademischen Sinne und nicht im Sinne einer bestimmten Tradition, z.B. der Wiener Schule, verstanden, sondern im Sinne eines umfassenden musikalischen Kulturgedächtnisses, das in jedem neuen Werk in neuer Beleuchtung aktiviert wird. Das erinnert an Verfahren des russischen Akmeismus, wie sie Renate Lachmann beschreibt:

»Die fremden Texte, sofern sie durch Strategien des Zitats, der Allusion, der Syllepse, des Anagramms etc. in den gegebenen Text eingebracht werden, begründen die Heterogenität des akmeistischen Textes. Die Heterogenität, die zugleich die Vorstellung der Kopräsenz der Texte abbildet, bricht nun aber den Einzeltext auf. Das heißt, im Verweis auf die fremden Texte überschreitet der Text seine eigenen Grenzen und

56 Nr. 157 der Sammlung Melodje ludowe litewskie von A. Juszkiewicz.

57 Siehe hierzu Walsh, Stephen: The Shaman, the Sage, and the Sacrificial Victim - und Griffiths, Paul: Remembering »The Rite of Spring « ot »Ce que je n'ai pas voulu exprimer dans »Le Sacre du printemps in Danuser, Hermann u.a. [Hg.]: Avatar of Modernity. The right of spring reconsidered.

58 Boulez, Pierre/Sacher, Paul/Schuh, Oskar Fritz: »Persönlichkeit und Erscheinung. Gesprächsrunde zum 80. Geburtstag«, in: Lindlar, Heinrich: Igor Strawinsky, Frankfurt: Suhrkamp 1982, S. 155-164, Zitat 159. 
öffnet sich in den Text der Kultur, den Makrotext. [...] So entwickelt der akmeistische Text einen eigenen »Chronotop«, der die geschichtliche Zeit und ihre Markierungen dementiert. $\ll 99$

Schon 1910 schrieb Andrej Belyi: »Das Neue an der Gegenwartskunst besteht lediglich in der erdrückenden Masse alles Vergangenen, das auf einmal vor uns hochgeschwemmt wird; wir erleben im Augenblick alle Jahrhunderte und alle Nationen; das Leben der Vergangenheit zieht an uns vorüber. ${ }^{60}$ So wie Ossip Mandelštam von »Sehnsucht nach der Weltkultur ${ }^{61}$ war Strawinsky vom Wunsch getrieben, »alles was er liebt, sich zu eigen $\mathrm{zu}$ mache ${ }^{62}$ Es ist vollkommen evident und gegen Strawinskys eigenes Urteil zu betonen, dass die Werke von Le Sacre bis Les Noces einen von der musikalischen russischen Volkskunst bestimmten Gedächtnisraum realisieren. Sein Sohn, der Maler Theodore Strawinsky, bringt diesen Zug mit der Erfahrung des Exils ab 1914 in Verbindung: »Ein verständliches Heimweh erklärt wohl hinlänglich, warum Strawinsky damals so sehr für die russische Volkskunst schwärmte, deren unerschöpflichen Reichtum er liebevoll erforschte und auswertete. Diese Volkskunst lag ihm wahrhaft am Herzen. ${ }^{63}$

59 Lachmann, Renate: »Text und Gedächtnis. Bemerkungen zur Kulturosophie des Akmeismus«, in: Gumbrecht, Hans-Ulrich/Link-Heer, Ursula: Epochenschwellen und Epochenstrukturen im Diskurs der Literatur - und Sprachhistorie, Frankfurt: Suhrkamp 1985, S. 283-299, Zitat S. 287. Der Akmeismus entsteht um 1910 und endet 1966 mit dem Tod von Anna Achmatova.

60 Belyi, Andrej: Simvolizm, Moskau: 1910, zitiert nach R. Lachmann: Gedächtnis und Literatur, S. 87 Anm. 57.

61 Ebd., S. 286.

62 P. Boulez in Boulez, Pierre/Sacher, Paul/Schuh, Oskar Fritz: »Persönlichkeit und Erscheinung. Gesprächsrunde zum 80. Geburtstag«, in: Lindlar, Heinrich: Igor Strawinsky, Frankfurt a.M.: Suhrkamp 1982, S. 155-164, Zitat 159.

63 Strawinsky, Theodor: "Von Kindheit und Kriegszeit«, in: Lindlar, Heinrich: Igor Strawinsky, Frankfurt, Suhrkamp, S. 49-54, Zitat S. 53. 


\section{LE SACRE DU PRINTEMPS UND DAS KULTURELLE GEDÄCHTNIS}

Drittens nun - und diese Gedächtnis-Beziehung hat das musikalische Kunstwerk mit allen anderen Arten von Kunst gemeinsam - hat ein $\mathrm{Mu}-$ sikwerk die Chance, in das kulturelle Gedächtnis eines Landes, einer Kultur oder gar einer globalisierten Kulturgemeinschaft einzugehen. Was die Bedeutung eines Musikstïcks im kulturellen Gedächtnis angeht, muss man zwischen >Kanon< und >Repertoire < unterscheiden. Zum Repertoire gehört ein Stïck aufgrund seiner Beliebtheit, in den Kanon dagegen gelangt es durch intertextuelle Bezugnahmen in anderen Musikstücken und vor allem einem kritischen, kommentierenden und didaktischen Diskurs, der sich um es herum entfaltet. Kaum ein Musikwerk des 20. Jahrhunderts ist intensiver diskutiert und kommentiert worden als Le sacre du printemps. Kaum ein Werk hat einen größeren Einfluss auf andere Komponisten ausgeübt, kaum einem anderen Werk wird seit langem und bis heute ein vergleichbarer Rang eines musikgeschichtlichen Meilensteins zuerkannt.

In seiner Bedeutung als Wendepunkt der Kunst und Meilenstein der Moderne ist Strawinskys Ballett nur mit Picassos Demoiselles d'Avignon (1907) zu vergleichen. Beiden Werken gemeinsam ist der Primitivismus: die Verwendung archaischer Urformen, bei Picasso die afrikanischen Masken anstelle der Gesichter bei einigen Figuren, bei Strawinsky die Dominanz stampfender irregulärer Rhythmen und der Wiederholung »rudimentärer Tonfolgen« (Adorno). Gemeinsam ist beiden auch der lange, sich in zahllosen Skizzen niederschlagende Anlauf, den beide zu ihren Werken genommen haben (zu Picassos Bild existieren ca. 800 Vorstudien $^{64,} \mathrm{zu}$ Strawinskys Partitur die unschätzbaren Skizzen). Schon dieser lange Anlauf zeigt, dass beide es auf einen weiten Sprung, einen revolutionierenden Durchbruch in künstlerisches Neuland angelegt haben. Entscheidend für die Kanonisierung des Sacre ist vor allem die Privilegierung des Neuen, $\mathrm{Zu}$ kunftsweisenden, Epochemachenden, die ein Element des modernen Zeit-

64 Küster, Bärbel: Die Skizzenbücher zu den »Demoiselles d'Avignon« und Picassos Auseinandersetzung mit prähistorischer Kunst, Magisterarbeit, Universität Hamburg 1994. 
bewusstseins, des »Zeitregimes der Moderne « ist. ${ }^{65}$ Im Horizont dieses spezifisch modernen Paradigmas sind es die Vorläufer, Wegbereiter, Bahnbrecher, denen das Interesse gilt; anders ausgedrückt, sind es gerade diese Aspekte der kanonisierten Werke, denen sie ihre Aufnahme in den Kanon verdanken. In dieser Perspektive ist es gerade der Skandal, den der Sacre bei seiner Uraufführung am 29. Mai 1913 im Thêatre des Champs-Élysées auslöste, der diesem Stück den Stempel revolutionärer Neuheit aufdrückte und es sofort als epochemachend heraushob, während normalerweise der Rang des Epochemachenden einem Werk erst im Nachhinein, im Rahmen des sich erst nach einigen Dezennien konstituierenden kulturellen Gedächtnisses zuwächst.

In diesem Zusammenhang ist nun das Urteil eines Kenners wie Adorno umso merkwürdiger, der gerade den Sacre, diesen Inbegriff des Unerhörten, schockierend Neuen in der Musikgeschichte, als »Regression« und »Infantilismus « abkanzelte. ${ }^{66}$ Der Fall Adorno beleuchtet beispielhaft die normativen Ansprüche der ästhetischen Modernisierungstheorie, ihres Fortschrittsglaubens und ihres absoluten Innovationsgebots. Seine Philosophie der neuen Musik gliedert sich in die beiden Teile >Schönberg und der Fortschritt< und >Strawinsky und die Restauration< - eine Antithese, von der Schönberg selbst bekanntlich nichts wissen wollte. Das Prinzip des musikalischen Fortschritts definiert Adorno vollkommen überzeugend negativ: als einen »Kanon des Verbotenen«; musikalischer Fortschritt manifestiert sich in der Inkriminierung des Überholten. „Wenn nicht alles trügt«, schrieb Adorno in der Vorrede 1948, »schließt er [der Kanon des Verbotenen] heute bereits die Mittel der Tonalität, also die der gesamten traditionellen Musik, aus. ${ }^{67}$ Das Überholte wird nicht nur normativ ausge-

65 Siehe hierzu Assmann, Aleida: Ist die Zeit aus den Fugen? Aufstieg und Fall des Zeitregimes der Moderne, München: C.H. Beck 2013, besonders S. 132-183.

66 Allerdings gehörte Infantilismus, wie.Ingold betont, zum Programm des russischen Neoprimitivismus: »Die >Jugendlichkeit< der archaisierenden Neuerer, ihr Bestehen auf kindlicher Frische und Unvoreingenommenheit, aber auch ihre gewollt kindischen Ausdrucks- und Verhaltensformen gehören zur Gruppenästhetik des Neoprimitivismus, der in Russland um 1913 den Höhepunkt seiner künstlerischen Entwicklung erreichte« P.F. Ingold: Der große Bruch, S. 181.

67 Th. W. Adorno, Philosophie der neuen Musik, S. 40 vgl. Thomas Mann, Doktor Faustus, S. 349 »Jeder Bessere trägt in sich einen Kanon des Verbotenen, des 
schlossen, es wird geradezu zum Gegenstand des Abscheus, der Abjektion im Sinne Julia Kristevas ${ }^{68}$, wie es etwa Thomas Mann in seinen Josephsromanen als das religiös »Überständige « beschrieben hat. ${ }^{69}$ Adornos Verdikt des in seinen Augen musikalisch Überholten erinnert in seiner persekutorischen Schärfe an das biblische Bilderverbot. Im Licht dieser normativen Kunstreligion und ihrer Verbote erscheint der Sacre und vor allem Strawinskys neoklassisches Euvre als Regression in den Götzendienst, als Tanz um das Goldene Kalb. ${ }^{70}$ Das Großartige von Strawinskys Musik liegt darin, ihre Neuheit nicht mit den Dogmen und Verboten eines normativen Modernismus umstellt, sondern sich Freiheiten genommen zu haben, die auch diese Verbote über den Haufen warfen. Heute, wo dieser >Kanon des Verbotenen< längst verblasst ist, hat Strawinskys Sacre einen zentralen, bleibenden und vollkommen unbezweifelten Platz im kulturellen Gedächtnis als das vielleicht berühmteste Werk der musikalischen Moderne.

Sich-verbietenden, der nachgerade die Mittel der Tonalität, also aller traditionellen Musik umfasst.«

68 Kristeva, Julia: Les pouvoirs de l'horreur. Essai sur l'abjection, Paris: Éditions du Seuil 1980.

69 "Gewisse Dinge«, erklärt Thomas Mann in einem Brief diese Konzeption, »waren einmal ganz richtig und vernünftig, hören aber auf, es zu sein und werden zur >Gottesdummheit<. Religiosität besteht wesentlich darin, hierauf, auf Veränderungen im Bilde der Wahrheit und des Rechten achtzugeben.« (in: Thomas Mann Selbstkommentare: »Joseph und seine Brüder«, hg. von Hans Wysling und Marianne Eich- Fischer, Frankfurt 1999, 292f). Dasselbe Prinzip illustriert Adorno am Beispiel des verminderten Septimakkords: Er »ist richtig und allen Ausdrucks voll am Beginn von Beethovens Sonate op. 111 « und »klingt falsch in den Salonpiècen« (Th. W. Adorno, Philosophie der neuen Musik, S. 40f.), d.h. ist zum Inbegriff musikalischen Kitschs geworden.

70 Schönbergs Komposition dieses Tanzes in seiner Oper Moses und Aron - auch eine musikalische Vergegenwärtigung des Heidentums - spielt denn auch unüberhörbar auf den Sacre an. 